\title{
Laparoscopic left hemihepatectomy in small dogs: an easy and effective new technique
}

\author{
Hua Zhang ${ }^{1,2}$, Jing Wang ${ }^{1}$, Yaqin $\mathrm{Cao}^{1}$, Zhao nan Zhang ${ }^{1}$, Jing Shen ${ }^{1}$, \\ Jinjin Tong ${ }^{2}$, Hong bin Wang $^{3}$
}

${ }^{1}$ Beijing University of Agriculture, Animal Science and Technology College, Beijing Key Laboratory of Traditional Chinese Veterinary Medicine, Beijing, China

${ }^{2}$ Beijing University of Agriculture, Animal Science and Technology College, Beijing Key Laboratory of Dairy Cow Nutrition, Beijing, China

${ }^{3}$ Northeast Agricultural University, College of Veterinary Medicine, Department of Veterinary Surgery, Harbin, China

Received January 1, 2020

Accepted December 21, 2020

\begin{abstract}
The present report describes a novel approach to and presents the surgical results for laparoscopic hepatectomy of the left lobes in small dogs. A purely laparoscopic four-port approach was used in seven beagles. The left triangular ligament was cut first, then the base of the left medial lobe (LMB) was ligated with silk thread using a needle, and the liver parenchyma of the LMB approximately $0.5 \mathrm{~cm}$ above the ligation site was removed with LigaSure. The left lateral lobe (LLB) was removed in the same manner. Blood was collected on postoperative days $1,3,7$, and 14 for laboratory testing. Left hepatic lobe resection was successfully completed in all dogs, with an average operation time of $102.53 \pm 9.07 \mathrm{~min}$ and an average blood loss of $32.10 \pm 6.43 \mathrm{ml}$. Serious postoperative complications were not observed. The white blood cell (WBC) count and alkaline phosphatase (ALP) level were significantly increased in experimental dogs 1 day after surgery $(P<0.05)$; aspartate transferase (AST) and alanine transaminase (ALT) were significantly different before and after surgery $(P<0.01)$; and all the indicators were basically normal seven days after the operation. Exploratory laparoscopy at 28 days revealed adhesion of the surface of the liver section to the omentum or the gastric wall, but there were no other abnormal findings. The combined application of liver pedicle ligation and LigaSure for liver lobectomy provides a simple and effective method for generating liver lobectomy models for research on liver disease and regeneration and a feasible laparoscopic liver lobectomy operation scheme for small dogs $(10-15 \mathrm{~kg})$.
\end{abstract}

Hepatectomy, beagle, laparoscopy, animal model

Hepatectomy is widely used in medical research and experimental surgery, for studying liver failure (Imai et al. 2019; Meyer et al. 2019; Shirata et al. 2019), regeneration (Schadde et al. 2017; Tsai et al. 2018), and transplantation (Etheredge et al. 2019; Lué et al. 2019; Majd et al. 2019), and is one of the common hepatic procedures performed on companion animals (Boothe 2015; Zhang et al. 2014). Thus, an animal model of hepatectomy is an extremely important research tool for evaluating liver regeneration after various treatments (Bernat Córdoba-Jover 2019; de Miguel et al. 2019; Lee et al. 2019). The successful establishment of hepatectomy models could promote technological advances and the development of new methods for the treatment of hepatic diseases.

Laparoscopic techniques have many advantages, such as a smaller wound, a faster return to full activity, and less pain, effectively reducing the adverse effects of surgical stress. Laparoscopic methods can be used to establish experimental animal models as well as to perform liver surgery (Chen et al. 2018; Liu et al. 2019; Takahara et al. 2016; Zhang et al. 2014a). However, laparoscopic liver surgery remains technically challenging due to the greater risk of bleeding. Compared to human medicine, laparoscopic hepatectomy is rarely used in veterinary surgery, mostly because of the expense of the instruments and the complexity of the procedure (Yoshida et al. 2019; Zhang et al. 2014b). Beagles are

Address for correspondence:

Jinjin Tong, Hong-bin Wang

Beijing University of Agriculture, Beijing

Northeast Agricultural University, Harbin

People's Republic of China 
important experimental research animals because their immune responses and metabolic pathways are similar to those of humans, and they are relatively easy to manage and perform procedures on (Ferreira et al. 2019; Frezza and Wachtel 2006; Tao et al. 2016). Therefore, in this study, we selected beagles as the experimental surgical model for developing and testing a simple and effective new method for liver lobectomy, and to provide a reference for the establishment of animal models for research on treatment of liver disease in small animals.

\section{Materials and Methods}

\section{Ethical standards}

The experimental protocols were approved by the Animal Ethics Committee of the Beijing University of Agriculture (BUAEC2019-0205). All procedures performed in the studies involving animals were in accordance with the Guide for the Care and Use of Laboratory Animals of the National Research Council.

Animals

Seven female beagle dogs at a mean age of one year (10-15 months) and mean body weight of $13.10 \pm 1.55 \mathrm{~kg}$ (range 10.8-15.2 kg) were purchased from the Beijing Marshall Biotechnology Co., Ltd., China (No. SYXK2019-0047). The experimental protocol was approved by the Animal Ethics Committee of the Beijing University of Agriculture (BUAEC 2019-0205). Animal care and handling were performed in accordance with the Guide for the Care and Use of Laboratory Animals of the National Research Council. The dogs were housed individually and fed a standard diet (Beijing Keao Xieli Feed Co., Ltd., China) with ad libitum access to tap water.

\section{Anaesthetic protocol}

Before surgery, all dogs were fasted for $12 \mathrm{~h}$, and water was withheld for $6 \mathrm{~h}$. The dogs were premedicated with an intramuscular injection of atropine sulphate $(0.05 \mathrm{mg} / \mathrm{kg}$ body weight; Shanghai Pharmaceutical Group, China), ketamine $(20 \mathrm{mg} / \mathrm{kg}$ body weight, Chemfun Medical Technology, China) and acepromazine $(0.1 \quad \mathrm{mg} / \mathrm{kg}$ body weight, Janssen Pharmaceutical, China). A cephalic vein of the forelimb was cannulated for further induction and fluid administration. General anaesthesia was induced by an intravenous injection of $2-3 \mathrm{mg} / \mathrm{kg}$ body weight propofol ( $1 \%$ propofol-MCT/LCT, Jiabo Pharmaceutical, China), followed by endotracheal intubation. Anaesthesia was maintained with $1-2 \%$ isoflurane, the respiratory rate of the anaesthesia machine was adjusted to $13 \times / \mathrm{min}$, and the tidal volume was adjusted to $10-15 \mathrm{ml} / \mathrm{kg}$. Anterior limb veins were injected with $0.9 \% \mathrm{NaCl}$ solution to maintain circulation. The operating room temperature was maintained at $25^{\circ} \mathrm{C}$. Vital signs including the heart rate, electrocardiogram, noninvasive arterial blood pressure, pulse oxygen saturation, respiratory rate, end-tidal carbon dioxide, and rectal temperature were measured continuously with a Philips monitor (Philips IntelliVue MP50; Philips Medizin Systeme Boeblingen, Germany).

\section{Surgical procedure}

The animals were placed in the supine position. The operation was performed under sterile conditions. The abdominal cavity was filled with carbon dioxide at a pressure of $10 \mathrm{~mm} \mathrm{Hg}$ by Veress needle to establish a pneumoperitoneum. Four laparoscopic port sheaths were placed through the abdominal wall: port A provided laparoscope access, and the other three ports (B, C, and D) provided access for surgical instruments (Fig. 1).

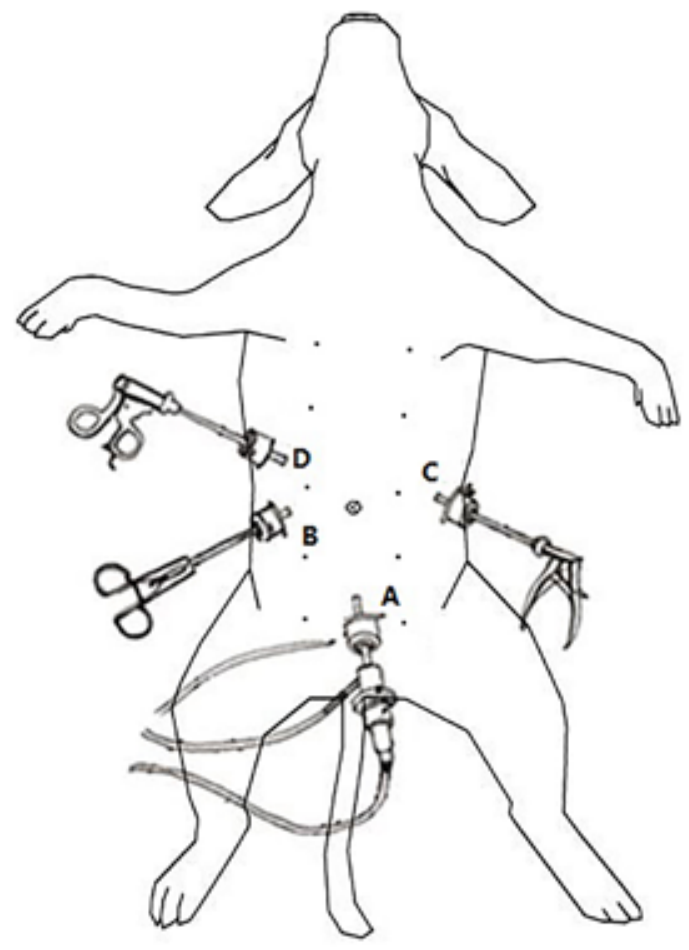

Fig. 1. Cannula locations. 
Port A, located 2-3 cm in front of the last pair of nipples along the ventral midline was used to insert the laparoscope. Port B was located $7.5-8.0 \mathrm{~cm}$ to the right of the umbilicus. A $5.0-5.5 \mathrm{~mm}$ trocar-cannula unit was used to insert dissecting or grasping forceps. Port $\mathrm{C}$ was located $7.5-8.0 \mathrm{~cm}$ to the left of the umbilicus; a 10.0-10.5 mm trocar-cannula unit was used to insert the LigaSure Atlas (LS1037,10 mm-37cm, Covidien Medical), a titanium clip applicator (10 mm diameter, Guangdian Medical Instruments, China), scissors (5 mm diameter, Guangdian Medical Instruments, China), needle-holding forceps ( $5 \mathrm{~mm}$ diameter, Guangdian Medical Instruments, China), large-jaw toothless grasping forceps (5 mm diameter, Guangdian Medical Instruments, China), a monopolar hook-electrode (5 mm diameter, Guangdian Medical Instruments, China) and a disposable specimen retrieval bag (Weide Medical Technology Co., Ltd., Hangzhou, China). Port D was 1-2 cm below the last costal arch on the right side of the abdominal wall, $9 \mathrm{~cm}$ from the midline, and a 5.0-5.5 mm trocar-cannula unit was used to insert grasping forceps.

The first step in the procedure was to use the LigaSure to remove the falciform ligaments. The left triangular ligament was cut, and the left lobe of the liver was completely dissociated (Plate III, Fig. 2a). The left medial lobe (LMB) was lifted with grasping forceps to expose the hilum of the liver. A needle with silk thread was used to ligate the LMB pedicle by an intracorporeal knotting technique (Plate III, Fig. 2b). Then, the liver parenchyma of the LMB $0.5 \mathrm{~cm}$ from the ligature site was removed with LigaSure (Plate III, Fig. 2c). Hepatic transection of the left lateral lobe (LLB) was performed in the same manner (Plate III, Fig. 2d). The raw surface was electrocoagulated with the unipolar electrocoagulation to stop errhysis (Plate III, Fig. 2e). No significant bleeding or bile leakage from the cut surface was noted after the saline rinse (Plate III, Fig. 2f). All resection surfaces of the liver were sealed with fibrin glue (Guangzhou Bioseal Biotechnology Co., Ltd, China) to ensure haemostasis and biliostasis. The resected lobes were then placed in a sterile bag and removed through an enlarged incision (4-5 cm) at port C. The abdominal cavity was irrigated and suctioned, and the abdominal incisions were closed.

Sampling and data collection

Operative data recorded at the time of surgery included the operation time, weight of the removed liver lobe, and final incision size. Blood loss was estimated by the amount of fluid collected in a suction container minus the amount of irrigation fluid. The diameter of the vessels at the raw surfaces (LMB and LLB) of the removed lobe after resection were also measured. The liver parenchyma at the raw surface was crushed using thumb and forefinger to expose the blood vessels, which were then laid out naturally on a glass plate. Vernier calipers were used to measure the half circumference of the blood vessel, and the formula, $r=c / 2 \pi$ ( $c$ is circumference, and $r$ is radius), was used to calculate the radius of the vessel.

Postoperative care

All animals were clinically examined daily, and complete blood count (CBC) analyses were performed. Blood was collected from all dogs before the surgery and on postoperative days (PODs) 1, 3, 7, and 14. Serum was separated to measure total bilirubin (T-Bil), aspartate transferase (AST), alanine transaminase (ALT), gammaglutamyl transferase $(\gamma-\mathrm{GT})$, albumin/globulin (A/G) and alkaline phosphatase (ALP). The follow-up laparoscopy was performed $28 \mathrm{~d}$ after surgery.

The physiological parameters of the animals (such as body temperature, heart rate, respiration rate and blood pressure) were closely monitored until they were restored to normal preoperative levels. Buprenorphine (0.01 mg/kg i.m. Hansen Pharma Co., Ltd., Changsha, China) and ampicillin (20 mg/kg i.m.; Lukang Pharma, Jining, China) were administered every $8 \mathrm{~h}$ for 3 days after surgery for analgesia and to prevent infection.

Statistical analysis

SPSS version 18.0 (SPSS Institute, Cary, NC, USA) was used for statistical analyses. One-way ANVOA was used to compare differences between preoperative and post-operative time points. The data are expressed as the mean $\pm \mathrm{SEM}$. A $P$ value of $<0.05$ indicated significance.

\section{Results}

In this study, seven laparoscopic left hemihepatectomies in dogs were successfully completed, with a mean operation time of $102.53 \pm 9.07 \mathrm{~min}$. The mean lobe mass was $183.18 \pm 37.53 \mathrm{~g}(\mathrm{LMB}=69.8 \pm 17.64 \mathrm{~g}, \mathrm{LLB}=146.63 \pm 34.19 \mathrm{~g})$, the total length of the surgical incision was $6.7 \pm 0.54 \mathrm{~cm}(6.0-7.5 \mathrm{~cm})$, the estimated blood loss was $32.10 \pm 6.43 \mathrm{ml}(10-50 \mathrm{ml})$. The diameters of the portal vein and the hepatic vein at theraw surface of the removed LMB were $5.02 \pm 0.89 \mathrm{~mm}(4.10-6.05 \mathrm{~mm})$, and $3.24 \pm 1.04 \mathrm{~mm}$ $(2.07-4.83 \mathrm{~mm})$; and $6.02 \pm 0.66 \mathrm{~mm}(5.11-7.22 \mathrm{~mm})$ and $4.39 \pm 1.08 \mathrm{~mm}(3.86-6.91 \mathrm{~mm})$, respectively, for the removed LLB.

All dogs had slight discomfort on the day of surgery. On POD 1, one dog vomited after eating, but the other dogs returned to normal. The sutures were removed ten days after 


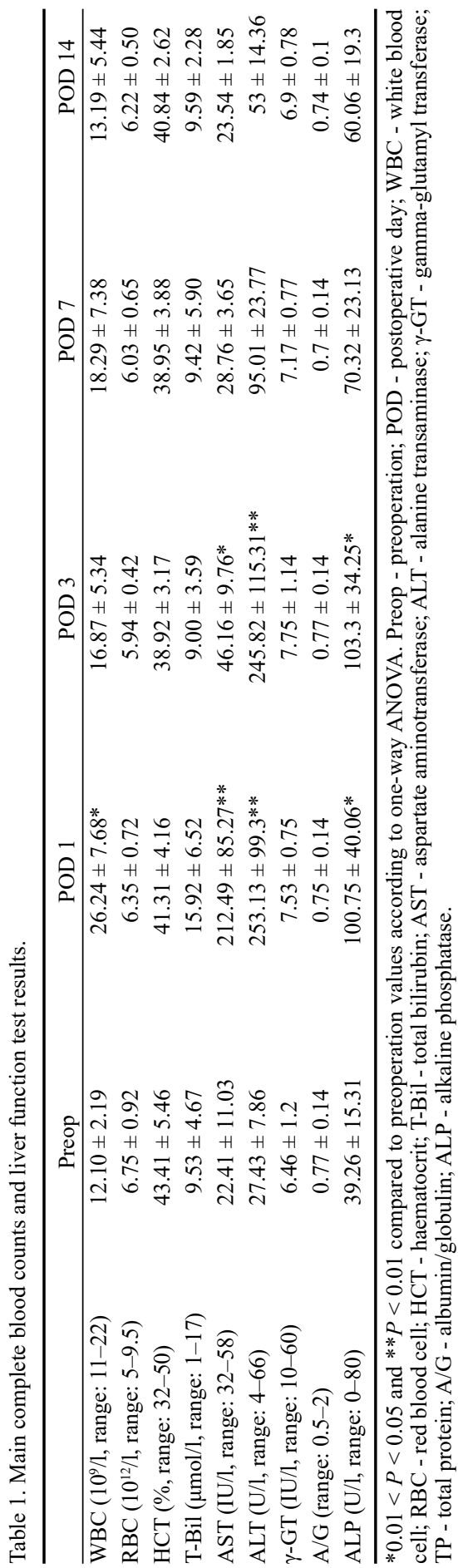

the operation, and the surgical incisions healed well in all animals without infection. White blood cell (WBC) count and ALP were significantly increased on POD 1 $(P<0.05)$. Alanine transaminase and AST were significantly higher at POD 1 than before surgery $(P<0.01)$, and all the indicators were basically normal seven days after the operation (Table 1). None of the dogs showed weight loss on POD 28. Exploratory laparoscopy was performed 28 day after surgery, and all findings were normal except for some adhesion of the raw surface of the left lobes to the omentum or gastric wall (Plate III, Fig 3).

\section{Discussion}

Currently, laparoscopic hepatectomy often requires both an experienced surgeon and expensive equipment, such as the Endo-GIA stapling system (Consten et al. 2008; Scuderi and Troisi 2014; Yao and $\mathrm{Wu}$ 2016), a saline-coupled bipolar sealer (Sastry et al. 2019), an ultrasonic scalpel (Chai et al. 2018; Kim et al. 2017), a laparoscopic laser (Ellebrecht et al. 2018) and a microwave applicator (Chong et al. 2018; Dimitri et al. 2019; Shen et al. 2018). These factors restrict the widespread use of hepatic lobectomy in veterinary clinics. In this study, we successfully performed left hemihepatectomy (LMB and LLB) in dogs by combining penetrating ligation at the base of the liver lobe with the squeeze and closure function of the LigaSure instrument to the liver parenchyma, which not only ensured safety but also simplified the surgical procedures and reduced difficulty.

The anatomy of the dog liver is different from that of the human liver. The canine hepatic artery branch is relatively thin and buried in connective tissue with the portal vein, making separation difficult and time-consuming. The confluence of the hepatic vein and posterior vena cava is in the liver parenchyma, and it is easy to tear these blood vessels during separation (Hasegawa et al. 2019). In humans, a fixation clip or tourniquet is often used to block blood flow in the 
hepatic artery, portal vein, and hepatic vein before ligation and transection of the liver parenchyma (Piardi et al. 2017; Yan et al. 2015). Some studies have proved that the traditional 'clamp-crush' technique can safely transect the liver parenchyma and block blood flow to the liver ( $\mathrm{Li}$ et al. 2018). Clinical practice has also proven that this method is relatively reliable in controlling bleeding. However, interrupting blood flow to the entire liver will cause ischaemia-reperfusion injury in the non-resected liver lobe, which is not conducive to postoperative recovery of the liver function (Yang et al. 2017); therefore, the above methods were evolved and improved in this study. During the operation, only the base of the resected liver lobe was treated by penetrating ligation to block blood flow into and out of the liver. Meanwhile, the LigaSure system was used to squeeze the liver parenchyma and seal the intrahepatic vessels, and the liver parenchyma separation was completed safely and quickly. LigaSure is a bipolar feedback-controlled sealing system that can seal blood vessels up to $0.7 \mathrm{~cm}$ (Saidi et al. 2009). LigaSure has been used in laparoscopic hepatectomy to effectively seal intrahepatic blood vessels and bile ducts without the need for separation (Yoshimoto et al. 2014). In this study, liver lobectomy was successfully completed with ligation of the base of the resected liver lobe before direct resection by LigaSure in beagles weighing 10-15 kg. According to a study on the anatomical structure of blood vessels in the dog liver, the branch portal veins of the LLB form the largest lobar portal vascular branch of the liver with a mean caliber of $0.42 \pm 0.08 \mathrm{~cm}(<15 \mathrm{~kg} \mathrm{dogs})$ or $0.60 \pm 0.06 \mathrm{~cm}(>15 \mathrm{~kg} \mathrm{dogs})$; the mean caliber of the largest hepatic vein was $0.50 \pm 0.11 \mathrm{~cm}(<15 \mathrm{~kg}$ dogs $)$ and $0.67 \pm 0.18 \mathrm{~cm}(>15 \mathrm{~kg} \operatorname{dogs})$ in the LBM and $0.60 \pm 0.06 \mathrm{~cm}(<15 \mathrm{~kg} \operatorname{dogs})$ and $0.82 \pm 0.10 \mathrm{~cm}(>15 \mathrm{~kg} \operatorname{dogs})$ in the LLB (Mari and Acocella 2015). In dogs weighing more than $15 \mathrm{~kg}$, the hepatic vein caliber may exceed $0.7 \mathrm{~cm}$, and LigaSure is unsafe for direct resection. Ligation of the liver pedicle before LigaSure is recommended to reduce the risk of bleeding.

To avoid gas embolization while still meeting the need for surgical space, the $\mathrm{CO}_{2}$ pneumoperitoneum pressure should not be too high during the operation; a pneumoperitoneum pressure of $8-10 \mathrm{~mm} \mathrm{Hg}$ is recommended (Machado et al. 2004; Constant et al. 2005; Frezza and Wachtel 2006). To better expose the liver, the falciform ligament should be removed. During removal of the falciform ligament, the surgical assistant may insert the grasp forceps in port D to pull and fix the falciform ligament, thus helping the surgeon. During the dissection of the left triangular ligament, the use of a monopolar hook electrode is not recommended because the triangular ligament is close to the diaphragm and a monopolar hook electrode can easily damage the diaphragm and cause pneumothorax. Good choices for cutting ligaments include scissors, ultrasonic dissectors and LigaSure, which has obvious advantages in operations close to the diaphragm.

Treating the remaining liver surfaces with electrocoagulation after lobectomy can prevent bleeding and bile leakage (Kim et al. 2017). When the LigaSure jaws are slightly opened and then placed on the raw surface of the remaining liver and activated, bleeding is stopped safely and effectively. The removed LLB, LMB, and falciform ligaments are placed in two separate specimen bags to make it easier to remove through the enlarged incision.

In conclusion, this report describes a minimally invasive surgical method for laparoscopic left hemihepatectomy in small dogs $(10-15 \mathrm{~kg})$ involving penetrating ligation to resect the liver lobe, squeezing the liver parenchyma and sealing the intrahepatic vessels with LigaSure. The postoperative outcomes demonstrate that this is a promising surgical procedure. This canine model could be useful for investigating liver disease and regeneration and could provide preclinical information for improving hepatobiliary surgical procedures in small dogs. This method of liver resection may be advantageous for smaller, more peripherally located lesions in LMB and LLB, such as hepatic abscessation, hepatic mass lesions (hepatocellular tumours, cholangiocellular tumours neuroendocrine tumours, mesenchymal tumours), and other liver conditions such as hydatid cyst, and haemangioma, 
but may not be appropriate for larger lesions in the LMB and LLB and for lesions involving the right lateral, right medial, caudate, or quadrate lobes (Boothe 2015; Machado et al. 2004). Further clinical studies are needed to evaluate and improve the efficacy of this surgical approach in the treatment of liver disease.

\section{Conflict of interest}

The authors have declared no conflicts of interest.

\section{Acknowledgements}

This study was funded by the National Natural Science Foundation of China (nos. 31702302 and 31802091 ), Da Bei Nong Foundation for Young Teachers (15ZK006).

\section{References}

Bernat Córdoba-Jover AAJR 2019: Cerium oxide nanoparticles improve liver regeneration after acetaminopheninduced liver injury and partial hepatectomy in rats. J Nanobiotechnology 17: 112

Boothe HJ 2015: Current concepts in hepatobiliary surgery. Vet Clin North Am Small Anim Pract 45: 463-475

Chai S, Zhao J, Zhang Y, Xiang S, Zhang W 2018: Arantius ligament suspension: a novel technique for retraction of the left lateral lobe liver during laparoscopic isolated caudate lobectomy. J Laparoendosc Adv Surg Tech A 28: 740-744

Chen K, Pan Y, Hu GY, Maher H, Zheng XY, Yan JF 2018: Laparoscopic versus open major hepatectomy for hepatocellular carcinoma: a meta-analysis. Surg Laparosc Endosc Percutan Tech 28: 267-274

Chong C, Lee KF, Chu CM, Chan A, Wong J, Chan SL, Lok HT, Fung A, Fong A, Cheung YS, Yu S, Johnson P, Lai P 2018: Microwave ablation provides better survival than liver resection for hepatocellular carcinoma in patients with borderline liver function: application of ALBI score to patient selection. HPB (Oxford) 20: $546-554$

Constant DL, Slakey DP, Campeau RJ, Dunne JB 2005: Laparoscopic nonanatomic hepatic resection employing the LigaSure device. JSLS 9: 35-38

Consten EC, Dakin GF, Robertus JL, Bardaro S, Milone L, Gagner M 2008: Perioperative outcome of laparoscopic left lateral liver resection is improved by using a bioabsorbable staple line reinforcement material in a porcine model. Surg Endosc 22: 1188-1193

de Miguel MP, Prieto I, Moratilla A, Arias J, Aller MA 2019: Mesenchymal stem cells for liver regeneration in liver failure: from experimental models to clinical trials. Stem Cells Int 2019: 3945672

Dimitri M, Staderini F, Brancadoro M, Frosini F, Coratti A, Capineri L, Corvi A, Cianchi F, Biffi GG 2019: A new microwave applicator for laparoscopic and robotic liver resection. Int J Hyperthermia 36: 75-86

Ellebrecht DB, Theisen-Kunde D, Kuempers C, Keck T, Kleemann M, Wolken H 2018: Analysis of laparoscopic laser liver resection in standardized porcine model. Surg Endosc 32: 4966-4972

Etheredge HR, Fabian J, Duncan M, Conradie F, Botha J 2019: Needs must: Living donor liver transplantation from an HIV-positive mother to her HIV-negative child in Johannesburg, South Africa. J Med Ethics 45: 287-290

Ferreira M, Salavati SS, Schoenebeck JJ, Clements DN, Campbell SM, Gaylor DE, Mellanby RJ, Gow AG, Salavati M 2019: Lactulose drives a reversible reduction and qualitative modulation of the faecal microbiota diversity in healthy dogs. Sci Rep 9: 13350

Frezza EE, Wachtel MS 2006: A proposed canine model of laparoscopic nonanatomic liver resection. J Laparoendosc Adv Surg Tech A 16: 15-20

Hasegawa Y, Nitta H, Takahara T, Katagiri H, Kanno S, Sasaki A 2019: Pure laparoscopic living donor hepatectomy using the Glissonean pedicle approach (with video). Surg Endosc 33: 2704-2709

Imai D, Maeda T, Wang H, Sanefuji K, Mori M 2019: Elevation of Mac-2 binding protein glycosylation isomer after hepatectomy is associated with post-hepatectomy liver failure, total Pringle time, and renal dysfunction. Ann Gastroenterol Surg 3: 515-522

Kim SH, Kim KH, Kirchner VA, Lee SK 2017: Pure laparoscopic right hepatectomy for giant hemangioma using anterior approach. Surg Endosc 31: 2338-2339

Lee GS, Yang HG, Kim JH, Ahn YM, Han MD, Kim WJ 2019: Pine (Pinus densiflora) needle extract could promote the expression of PCNA and Ki-67 after partial hepatectomy in rat. Acta Cir Bras 34: e201900606

Li YC, Chao A, Yang LY, Huang HY, Huang YT, Kuo HH, Wang CJ 2018: Electrothermal bipolar vessel sealing device (LigaSure) versus conventional diathermy in laparoscopic myomectomy: A propensity-matched analysis. Plos One 13: e193611

Liu Q, Liu F, Ding J, Wei Y, Li B 2019: Surgical outcomes and quality of life between laparoscopic and open approach for hepatic hemangioma: A propensity score matching analysis. Medicine (Baltimore) 98: e14485

Lué A, Martinez E, Navarro M, Laredo V, Lorente S, Jose Araiz J, Agustin Garcia-Gil F, Serrano MT 2019: Donor age predicts calcineurin inhibitor induced neurotoxicity after liver transplantation. Transplantation 103: e211-e215 
Machado MA, Galvão FH, Pompeu E, Ribeiro C, Bacchella T, Machado MC 2004: A canine model of laparoscopic segmental liver resection. J Laparoendosc Adv Surg Tech A 14: 325-328

Majd NE, Daneshi S, Hozani TT, Khajeh S, Razban V 2019: Decellularized liver transplant could be recellularized in rat partial hepatectomy model. J Biomed Mater Res A 107: 2576-2588

Mari L, Acocella F 2015: Vascular anatomy of canine hepatic venous system: a basis for liver surgery. Anat Histol Embryol 44: 212-224

Meyer J, Balaphas A, Combescure C, Morel P, Gonelle-Gispert C, Buhler L 2019: Systematic review and metaanalysis of thrombocytopenia as a predictor of post-hepatectomy liver failure. HPB (Oxford) 21: 1419-1426

Piardi T, Chetboun M, Cavallari S, Rhaiem R, Pessaux P, Kianmanesh R, Sommacale D 2017: Liver doubletightened maneuver: Optimal outflow control during liver parenchymal transection of the right and left hepatectomies. J Am Coll Surg 224: e11-e16

Saidi RF, Ahad A, Escobar R, Nalbantoglu I, Adsay V, Jacobs MJ 2009: Comparison between staple and vessel sealing device for parynchemal transection in laparoscopic liver surgery in a swine model. Hpb (Oxford) 9: 440-443

Sastry A, Sulzer JK, Passeri M, Baker EH, Vrochides D, McKillop IH, Iannitti DA, Martinie JB 2019: Efficacy of a laparoscopic saline-coupled bipolar sealer in minimally invasive hepatobiliary surgery. Surg Innov 26: 668-674

Schadde E, Hertl M, Breitenstein S, Beck-Schimmer B, Schl Pfer M 2017: Rat model of the associating liver partition and portal vein ligation for staged hepatectomy (ALPPS) procedure. J Vis Exp: 55895

Scuderi V, Troisi RI 2014: Tissue management with tri-staple technology in major and minor laparoscopic liver resections. Int Surg 99: 606-611

Shen H, Zhou S, Lou Y, Gao Y, Cao S, Wu D, Li G 2018: Microwave-assisted ablation improves the prognosis of patients with hepatocellular carcinoma undergoing liver resection. Technol Cancer Res Treat 17: 1077053628

Shirata C, Kokudo T, Arita J, Akamatsu N, Kaneko J, Sakamoto Y, Kokudo N, Hasegawa K 2019: AlbuminIndocyanine Green Evaluation (ALICE) grade combined with portal hypertension to predict posthepatectomy liver failure. Hepatol Res 49: 942-949

Takahara T, Wakabayashi G, Konno H, Gotoh M, Yamaue H, Yanaga K, Fujimoto J, Kaneko H, Unno M, Endo I, Seto Y, Miyata H, Miyazaki M, Yamamoto M 2016: Comparison of laparoscopic major hepatectomy with propensity score matched open cases from the National Clinical Database in Japan. J Hepatobiliary Pancreat Sci 23: 721-734

Tao Y, Liu Q, Jiang T, Wang X, Kang Y 2016: Channelled $\beta$-TCP scaffolds promoted vascularization and bone augmentation in mandible of beagle dogs. Adv Funct Mater 36: 6719-6727

Tsai JM, Weissman IL, Rinkevich Y 2018: Partial lobular hepatectomy: a surgical model for morphologic liver regeneration. J Vis Exp: 57302

Yan R, Li Y, Zhang L, Xia N, Liu Q, Sun H, Guo H 2015: Augmenter of liver regeneration attenuates inflammation of renal ischemia/reperfusion injury through the NF-kappa B pathway in rats. Int Urol Nephrol 47: 861-868

Yang X, Cao J, Yan Y, Liu F, Li T, Han L, Ye C, Zheng S, Wang S, Ye Y, Jiang K 2017: Comparison of the safety of electrotome, Harmonic scalpel, and LigaSure for management of thyroid surgery. Head Neck 39: 1078-1085

Yao DB, Wu SD 2016: Application of stapling devices in liver surgery: Current status and future prospects. World J Gastroenterol 22: 7091-7098

Yoshida H, Taniai N, Yoshioka M, Hirakata A, Kawano Y, Shimizu T, Ueda J, Takata H, Nakamura Y, Mamada Y 2019: Current status of laparoscopic hepatectomy. J Nippon Med Sch 86:201-206

Yoshimoto M, Endo K, Hanaki T, Watanabe J, Tokuyasu N, Sakamoto T, Honjo S, Hirooka Y, Ikeguchi M 2014: Effectiveness of the LigaSure small jaw vessel-sealing system in hepatic resection. Yonago Acta Med 57: 93-98

Zhang H, Liu T, Wang Y, Liu HF, Zhang JT, Wu YS, Lei L, Wang HB 2014a: Laparoscopic left hepatectomy in swine: a safe and feasible technique. J Vet Sci 15: 417-422

Zhang H, Zhou ZF, Zhang JT, Zhang SX, Wang HB 2014b: Laparoscopic cystostomy in pigs: Technique and comparison with traditional open cystostomy for surgical stress. Acta Vet Brno 83: 385-391 
Plate III

Zhang H. et al.: Laparoscopic ... pp. 367-373



Fig. 2. Intraoperative views. A: LigaSure dissected the left triangular ligament. B: Use of a needle with suture to ligate the base of the left medial lobe (LMB). C: LigaSure resected the LMB. D: LigaSure removed the liver parenchyma of the left lateral lobe (LLB). E: Resected liver surfaces treated by electrocoagulation. F: Image of the raw liver section.



Fig. 3. Follow-up laparoscopic examination at 28 days after surgery. 